\title{
Spontaneous Growth of GaN Nanowire Nuclei on N- and Al-polar AlN: A Piezoresponse Force Microscopy Study of Crystallographic Polarity ${ }^{*}$
}

\author{
Matt D. Brubaker, Alexana Roshko, Paul T. Blanchard, Todd E. Harvey, Norman A. \\ Sanford, and Kris A. Bertness \\ Physical Measurement Laboratory, National Institute of Standards and Technology \\ 325 Broadway, Boulder, CO 80305
}

\begin{abstract}
The polarity of gallium nitride $(\mathrm{GaN})$ nanowire nuclei grown on AIN layers was studied by piezoresponse force microscopy (PFM). N- or Al-polar AlN layers were grown by molecular beam epitaxy (MBE) on Si (111) substrates by use of Al- or N-rich growth conditions, respectively. Short and low density GaN nanowires were then grown on each AIN polarity type. PFM measurements verified the expected AIN layer polarity and further indicated that predominantly N-polar nanowires are produced for growth on both AlN polarity types. Crosssection scanning transmission electron microscopy (STEM) images further reveal that the nanowires on Al-polar AlN films are nucleated on regions in the AlN layer that contain inversion domains, which propagate into the GaN nanowire nuclei. PFM measurements were found to be a convenient technique for mapping the polarity of a statistically significant number of individual GaN nanowires.
\end{abstract}

Keywords: Gallium Nitride, Nanowires, Crystallographic Polarity, Piezoresponse Force Microscopy

The wurtzite crystal structure of GaN and related alloys is characterized by asymmetry along what is conventionally called the $c$-axis or (0001) direction of the crystal. The polarity of a given specimen refers to the relative orientation of this (0001) crystal direction with respect to the direction of growth. Crystallographic polarity has profound effects on the synthesis and performance of GaN-based devices and includes built-in polarization fields, ${ }^{1}$ polarity-dependent growth temperature limitations, ${ }^{2-4}$ incorporation of $\mathrm{p}$-type dopants ${ }^{5}$ (and compensating impurities $^{6}$ ), and performance of metal contacts. ${ }^{7,8}$ While many of these effects are now well established in planar $\mathrm{GaN}$ layers, the consequences for $\mathrm{GaN}$ nanowire ( $\mathrm{GaN} \mathrm{NW}$ ) growth and device performance are still under investigation. In particular, GaN NWs are often grown on silicon (111) substrates, which possess cubic symmetry and therefore do not present a template to guide the polarity of epitaxially grown wurtzite materials. There is no expectation for a particular NW polarity in this case and N-polar, Ga-polar, and mixed-polarity NWs have been reported variously throughout the literature.

Catalyst-free synthesis of gallium nitride nanowires is typically initiated through two distinct pathways - by templated growth with a masking layer or by spontaneous nucleation processes. In both cases, the crystallographic polarity of an underlying AlN nucleation layer has been shown to play a critical role in determining the structural characteristics of the nanowires. ${ }^{2,9-11}$ For templated nucleation using a masking layer, the polarity is usually propagated from the AlN layer into the GaN NW, producing NWs with dimensions that are largely determined by the

\footnotetext{
${ }^{*}$ Contribution of an agency of the U.S. government; not subject to copyright.
} 
etched features in the mask layer. ${ }^{2}$ However, for spontaneous nucleation on AlN surfaces, the GaN NWs do not necessarily exhibit the same polarity as surrounding regions and are often associated with inversion domains (IDs) in the AlN layer. These IDs have been reported to originate from eutectic Al-Si reactions for $\mathrm{Si}$ (111) substrates ${ }^{9,12}$ or from Si surface segregation in $\mathrm{SiC}$ substrates ${ }^{11}$ and can extend through the entire AlN layer.

As NWs may be nucleated by a variety of mechanisms which may be simultaneously active, the correlation of NW polarity with growth conditions should be determined from a large sample of individual NWs. In many studies, the NW polarity has been characterized by transmission electron microscope (TEM)-based methods using cross-section samples prepared as lamella. While TEM-based methods provide highly detailed information and excellent spatial resolution, they are time-consuming, low-throughput, and have practical limitations for the number of NWs that can be characterized. Increasingly, scanned probe techniques are being developed for NW polarity measurements that are convenient, can measure a statistically significant number of NWs on as-grown samples, and can provide imaging in an orthogonal direction to that of conventional TEM cross sections. ${ }^{9,13}$ By cross-correlating scanned probe and TEM measurements, which by themselves have limited capabilities for depth resolution, a more complete assessment of the NW polarity can be obtained. In this study, we utilize a scannedprobe-based technique, Piezoresponse Force Microscopy (PFM), which is capable of producing polarity surface maps of nanowire nuclei and requires minimal sample preparation.

Piezoresponse Force Microscopy (PFM) is a technique that was originally developed for investigating domain structure in ferroelectric thin films, ${ }^{14}$ but has also been applied to crystallographic polarity measurements in AlN and GaN layers. ${ }^{15-17}$ PFM measurements are based on the converse piezoelectric effect, where piezoelectric oscillations are induced by a sinusoidal voltage bias applied to the AFM tip and are detected in the cantilever deflection signal using a lock-in amplifier. We have previously utilized this technique to measure the polarity of AlN buffer layers used for spontaneous NW growth templates ${ }^{10}$ and to measure the polarity of $\mathrm{AlN}$ and AlN/GaN buffer layers for selective-area nanowire growth templates. ${ }^{2}$ In these studies, we consistently observed phase-polarity assignments that were inverted from those originally reported in the literature. ${ }^{15}$ The phase-polarity assignments in our work were experimentally determined for Al- and N-polar AlN by cross-correlating with other characterization techniques (polarity-sensitive etching and observation of polarity-dependent surface reconstructions by Reflection High Energy Electron Diffraction (RHEED)). ${ }^{2}$ Similarly, the phase-polarity assignments for GaN were determined by measurements of commercially available Ga-polar $\mathrm{GaN}$. In all cases, the experimentally derived phase-polarity assignments were shown to be consistent with the interpretations shown in Figure 1. When the applied field and the [0001] directions are parallel, as shown in Figure 1a for the positive tip bias cycle on N-polar material, there is a net vertical extension that lifts the cantilever and increases the defection, producing inphase behavior between the applied bias and measured deflection. Conversely, out-of-phase behavior is produced for the positive bias cycle when the applied field and [0001] directions are antiparallel, as for the Al-/Ga-polar material shown in Figure $1 \mathrm{~b}$. 

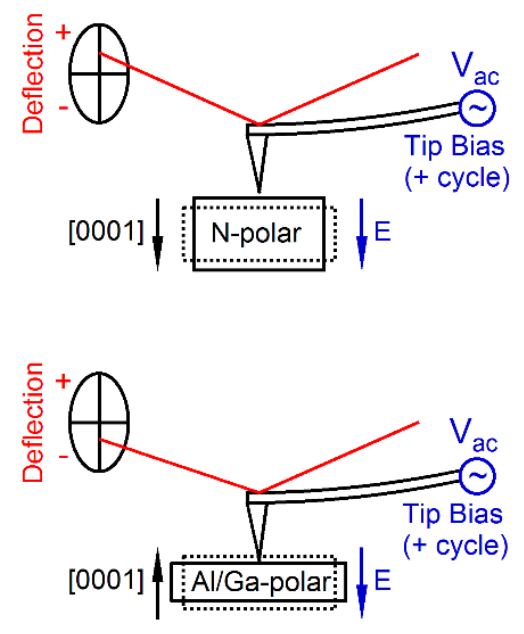

(a)

$$
\varepsilon_{3}=d_{33} \cdot E_{3}
$$$$
+++
$$

Tip Bias / Deflection ${ }^{+}{ }_{\text {In-phase }}^{+}$

(b)
FIG. 1. PFM polarity measurement and phase/polarity correlation for AlN or GaN with (a) N-polarity and (b) Al-/Gapolarity. Images shown are for the positive cycle of a sinusoidal tip bias.

In this study, we apply the PFM technique to evaluate the crystallographic polarity of spontaneously nucleated GaN NWs. Specifically, we grow short and low density GaN nanowire nuclei on both $\mathrm{N}$ - and Al-polar AlN surfaces and measure the polarity of the NWs and the adjacent AIN surfaces by PFM. The PFM phase/polarity is then superimposed against SEM data to map surface features and their polarity. Lastly, we supplement these results with crosssectional Scanning Transmission Electron Microscopy (STEM) measurements.

\section{Experimental Details}

Two GaN NW nuclei samples were grown on Si (111) substrates by molecular beam epitaxy (MBE), using equipment and procedures described elsewhere. ${ }^{2}$ In brief, the Si (111) substrates were $\mathrm{HF}$ cleaned and outgassed in the MBE system at $900{ }^{\circ} \mathrm{C}$. The substrate was then cooled to $790{ }^{\circ} \mathrm{C}$ and dosed with a few monolayers of $\mathrm{Al}$, prior to striking the nitrogen plasma. AlN was then grown for a duration of 24 minutes at a substrate temperature of $880{ }^{\circ} \mathrm{C}$. Finally, GaN was grown for 60 minutes, under N-rich conditions, and at a substrate temperature of $820^{\circ} \mathrm{C}$ to produce GaN NW nuclei. All temperatures were measured via backside optical pyrometry ${ }^{18}$, which detected the silicon $7 \times 7$ to $1 \times 1$ phase transition temperature at $820-830{ }^{\circ} \mathrm{C}$.

The AlN film polarity in each of the two samples was prescribed by use of N-or Al-rich growth conditions, which produces Al- or N-polarity, respectively. ${ }^{2}$ These N- and Al-rich growth conditions were attained by changing the Al cell temperature only, and RHEED images obtained during growth verified the intended growth condition and polarity, as discussed elsewhere in reference 2. For the Al-polar sample, a spotty RHEED pattern (indicative of N-rich growth conditions) with a $2 \times 6$ surface reconstruction (indicative of Al-polarity) was observed and is shown in Figures $2 a$ and $2 b$. A streaky $1 x 1$ pattern was observed for the N-polar sample (Figures $2 \mathrm{c}$ and $2 \mathrm{~d}$ ), which is indicative of the Al-rich growth conditions ${ }^{2,19}$. The total AlN thickness was approximately $84 \mathrm{~nm}$ for the Al-polar sample and $95 \mathrm{~nm}$ for the N-polar sample. 

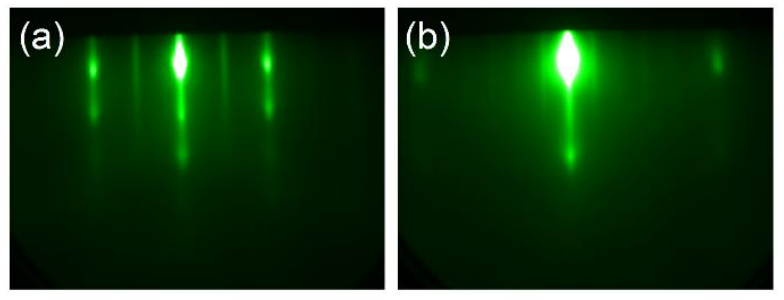

FIG. 2. RHEED images obtained during AlN buffer layer growth for $(a, b)$ Al-polar sample and (c,d) N-polar sample for electron beam azimuths in the $(\mathrm{a}, \mathrm{c})<11 \overline{2} 0>$ and $(\mathrm{b}, \mathrm{d})<10 \overline{1} 0>$ directions.
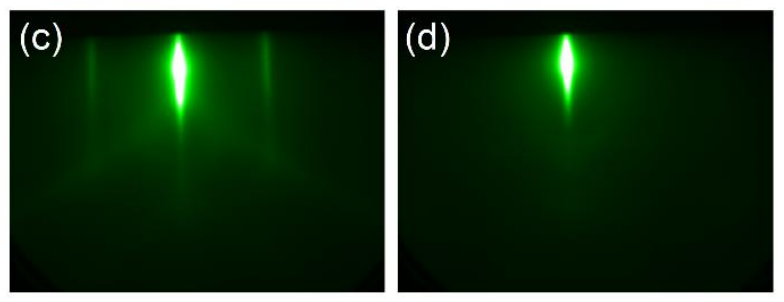

PFM measurements were then performed on each sample, simultaneously producing topography, PFM amplitude, and PFM phase images. Measurements were obtained using conductive-diamond-coated AFM tips with a nominal force constant of $20-80 \mathrm{~N} / \mathrm{m}$ and a nominal tip radius of $150 \mathrm{~nm}$. The AC bias (typically ranging from 3-5 $\mathrm{V}_{\mathrm{rms}}$ ) was applied to the tip and the sample substrate was held at ground potential. As the AFM tip radius is comparable to size of the nanowire nuclei under study (average diameter of approximately $50 \mathrm{~nm}$ ), the tip image artifact was substantial in the topography and PFM images; therefore, scanning electron microscope (SEM) images were also obtained for the PFM scan area. The PFM amplitude was uncalibrated with respect to the absolute deformation and is reported here as the amplitude of the lock-in detector signal. The PFM phase images were interpreted using the phase-polarity assignments described above. By superimposing the PFM phase on the SEM images, a composite image map showing detailed sample structure and local polarity was generated.

STEM images were also obtained for the Al-polar sample, which exhibited surface features with varying polarity, as will be discussed below. While PFM provides imaging of polarity variations in the surface plane, it does not indicate the depth at which polarity inversion occurs. Therefore, samples with polarity-inverted features were also studied by STEM in cross-section. For these measurements a lamella was cut from the sample using a focused ion beam (FIB) and ion milled to remove surface damage. Direct lattice imaging was then used to identify the polarity of specific features.

\section{Results and Discussion}

The PFM and SEM measurement results for the N-polar AIN sample are shown in Figure 3. As expected, the apparent size of the NWs in the topography image is substantially larger than the actual size shown in the SEM image, due to the large size of the probe tip in relation to the NW diameter. Nonetheless, the GaN NW nuclei are sufficiently short and isolated to allow the probe tip to contact both on the GaN NWs or on the exposed AlN buffer layer. The amplitude of the piezoelectric oscillations, shown in Figure 3c, is similar for both the GaN NW nuclei and the AlN layer. With the exception of a small area where the tip was not able to track the NW topography, the PFM measurement exhibited uniformly in-phase behavior. According to the phase-polarity assignments shown in Figure 1, the AlN regions possess N-polarity, which is consistent with the expected result for the Al-rich AlN growth mode. ${ }^{2}$ Similarly, the GaN NW 
nuclei also exhibit N-polarity, suggesting that the AlN layer polarity propagates into the GaN NW nuclei.

(a)
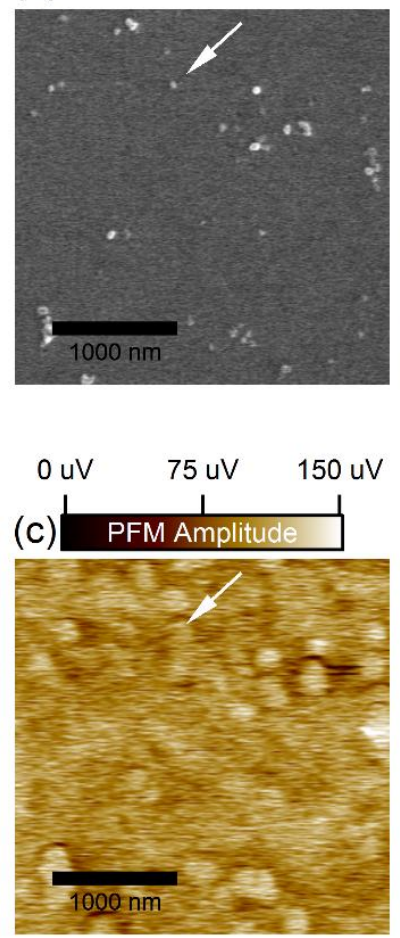

(e)

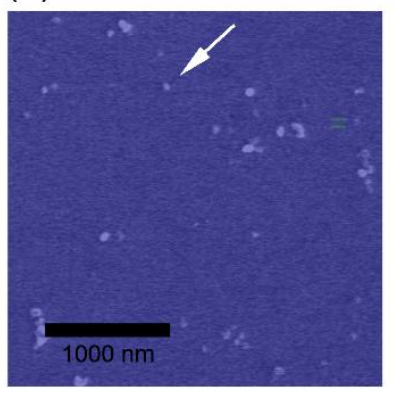

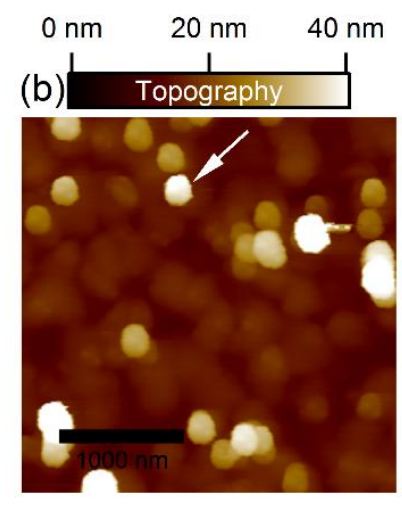
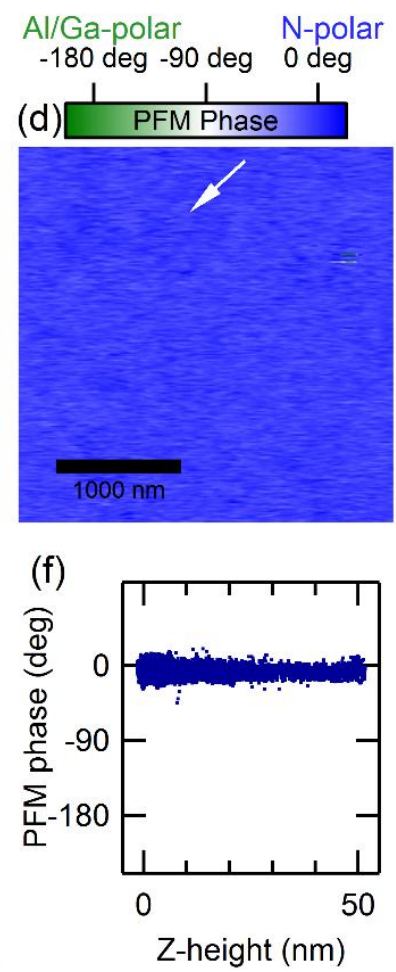

FIG. 3. Images of $\mathrm{GaN}$ nanowires grown on an N-polar AlN nucleation layer showing (a) SEM image of measurement area, (b) PFM topography, (c) PFM amplitude, and (d) PFM phase for a tip bias of $3 \mathrm{~V}_{\text {rms }}$. All images are from the same location. A composite $\mathrm{SEM} / \mathrm{PFM}$ phase image is shown in (e), where the Al-/Ga-polar or N-polar domains are indicated as green or blue overlay, respectively, are superimposed on the grayscale SEM image. An N-polar GaN nanowire surrounded by N-polar AlN is indicated by the arrow. The PFM phase data is correlated against the topography data in (f) and indicates uniform N-polarity.

The Al-polar AlN sample exhibits more complex PFM results than the uniform response of the N-polar AlN sample, as shown in Figure 4. As before, the tip exaggerates the diameter of the GaN NW nuclei, yet the AFM probe is still able to contact both GaN NWs and the surrounding AlN surface regions. An out-of-phase PFM signal is observed for the AlN surface regions, which indicates Al-polarity according to the phase-polarity conventions of Figure 1. These Alpolar AlN domains represent the dominant fraction of the surface area and are consistent with expectations based on the polarity-dependent surface reconstructions shown in Figures 2a and 2b. Interestingly, the GaN NW nuclei are N-polar and exhibit an in-phase behavior that is inverted from the surrounding AlN regions (example feature identified by solid arrow in Figure 4). It should again be noted that the apparent size of the N-polar domains is inflated in PFM 
phase image due to the AFM probe tip size - the polarity of the AlN domains immediately adjacent to the NW is uncertain as the probe does not contact these regions. The PFM amplitude of the GaN NWs is considerably lower than that of the surrounding AlN regions, as shown in Figure 4c. While a null in the PFM amplitude is expected at the boundaries between the $\mathrm{Al} / \mathrm{Ga}$ polar and N-polar regions, the PFM amplitude of the GaN NWs is suppressed in comparison to the sample grown on N-polar AlN (Figure 3c). It can be observed that in addition to the larger N-polar GaN NWs, there are also smaller GaN features in the SEM-PFM composite that are Gapolar (example feature identified by dashed arrow in Figure 4e). As shown in Figure 4f, these Ga-polar features do not grow to the same height as the N-polar NWs. While it is plausible that these small features are Ga-polar NW nuclei, it is more likely that they are the precursors of an emerging Ga-polar GaN matrix or compact layer that is often observed to grow between spontaneously nucleated NWs. ${ }^{11}$

(a)
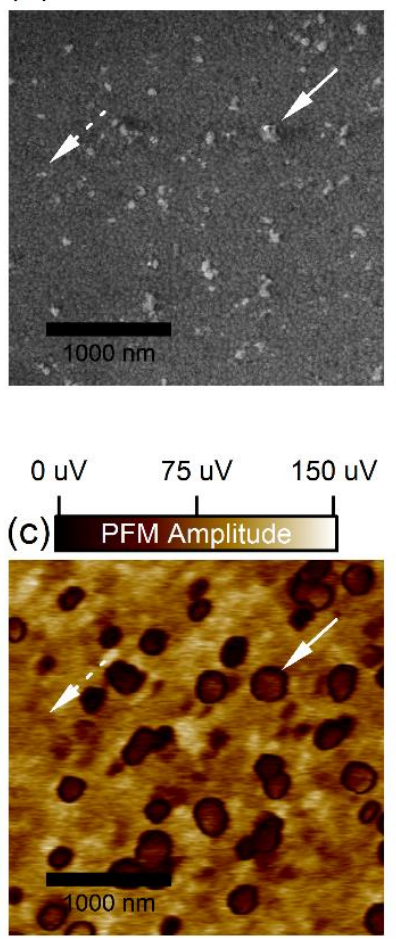

(e)

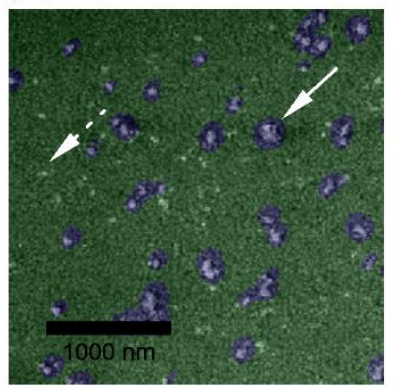

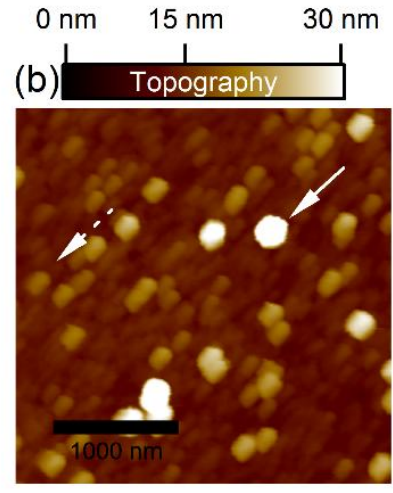

$\begin{array}{ll}\text { Al/Ga-polar } & \text { N-polar } \\ -180 \text { deg }-90 \text { deg } & 0 \text { deg }\end{array}$

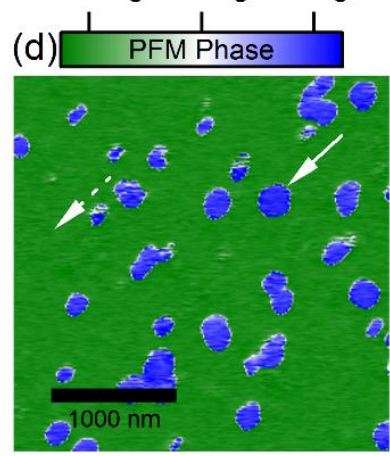

(f)

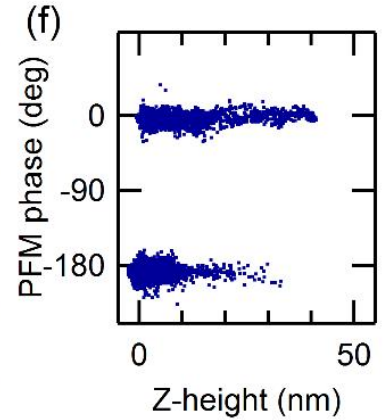

FIG. 4. Images of $\mathrm{GaN}$ nanowires grown on an Al-polar AlN nucleation layer showing (a) SEM image of measurement area, (b) PFM topography, (c) PFM amplitude, and (d) PFM phase for a tip bias of $5 \mathrm{~V}_{\text {rms. }}$. All images are from the same location. A composite $\mathrm{SEM} / \mathrm{PFM}$ phase image is shown in (e), where the Al-/Ga-polar or N-polar domains are indicated as green or blue overlay, respectively, are superimposed on the grayscale SEM image. An N-polar GaN nanowire surrounded by Alpolar AlN is indicated by the solid arrow and a smaller feature with Ga-polarity is indicated by the dashed arrow. The PFM phase data is correlated against the topography data in (f), which exhibits a clear distinction between the Al-/Ga- polar and N-polar regions. The out-ofphase Al-polar regions are primarily located at low z-height values that correspond to the AlN surface, while the in-phase $\mathrm{N}$-polar regions are also observed at larger z-heights corresponding to the GaN NWs. 
To investigate the source of the polarity inversion in the N-polar GaN NWs grown on Alpolar AIN, we have performed cross-section STEM imaging. Surprisingly, a thin section of Npolar AlN was observed at the $\mathrm{Si} / \mathrm{AlN}$ interface, as shown in Figure 5. For most of the sample, the top half of the AlN layer inverts to Al-polarity and is sparsely covered with Ga-polar GaN matrix material. However, in the subsurface vicinity of the GaN NW shown at left, the AlN is not fully inverted to Al-polarity. Instead, numerous vertical polarity inversion domains (identified by use of direct lattice images, details to be published elsewhere) were found to nucleate abruptly in the AIN and extend through the GaN NW nuclei. Of the two GaN NW nuclei that were examined in this fashion, both were observed to reside on AlN with a high density of inversion domains.

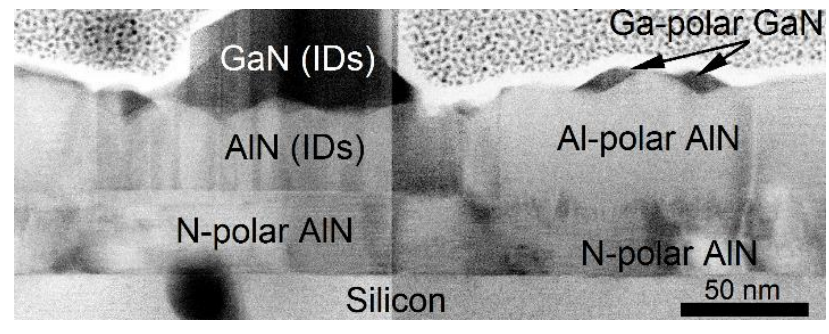

FIG. 5. STEM cross-section image of GaN NW sample grown on Al-polar AlN. Two images, obtained along the $<11 \overline{2} 0>$ zone axis, were stitched together to make the composite image. The polarity of various regions, as determined by direct lattice imaging, are indicated by text overlays and regions with inversion domains are labelled as IDs. The black spot near the silicon/AIN interface is a measurement-related contamination spot.

The interfacial N-polar section in the AlN layer, which was observed throughout the sample, may have resulted from evolving growth conditions. It is possible that a reduced $\mathrm{Al}$ desorption rate was produced during the temperature ramp from $790{ }^{\circ} \mathrm{C}$ (during $\mathrm{Al}$ pre-layer) to $880{ }^{\circ} \mathrm{C}$ (during the AlN layer) and resulted in temporarily Al-rich growth conditions, which are known to produce N-polar AlN. While the plasma conditions were not intentionally changed during the AlN layer, it is possible that the plasma source had not yet reached the equilibrium active nitrogen flux, also effectively producing temporary Al-rich growth conditions.

Using the combined results from STEM and PFM images a more complete picture of spontaneous NW nucleation on Al-polar AlN emerges. From the STEM images, the NWs appear to incorporate a partial fraction of N-polarity through inversion domains nucleated partway through the AIN layer. While this was only confirmed in two NWs by STEM, the large number of N-polar NWs observed in the PFM images suggests that this type of nucleation scenario can be generalized to other NWs in this sample. The relative volume of $\mathrm{N}$ - and Gapolar material in the NWs is difficult to assess in the STEM cross sections, due to projection effects though multiple domains in the lamella. For PFM measurements these inversion domains will tend to clamp the piezoelectric response, which is the probable explanation of the reduced PFM amplitude of the NWs shown in Figure 4c. However, the overall PFM response is likely a volume-averaged assessment of the GaN NW polarity and suggests that the NWs are predominantly $\mathrm{N}$-polar. It is possible that the interfacial N-polar layer at the $\mathrm{Si} / \mathrm{AlN}$ interface also contributes to the N-polar PFM response, however PFM measurements are typically more sensitive to the surface due to electric field concentration near the sharp tip ${ }^{14}$. As such, the PFM measurement may be less sensitive to the N-polar interfacial layer. These results indicate that N- 
polarity, either in the form of a homogeneous N-polar AIN surface or an inversion domain in an otherwise Al-polar AlN surface, is a key feature in the spontaneous nucleation of GaN NWs. The predominantly N-polar composition in these NWs is consistent with the generally-reported N-polarity in spontaneously nucleated GaN NWs. ${ }^{11,20,21}$

\section{Conclusions}

The polarity of GaN NWs, spontaneously nucleated on N- and Al-polar AlN surfaces, was studied by PFM. For N-polar AIN samples, both the GaN NW nuclei and the surrounding AlN surface regions were found to possess the same N-polarity. In contrast, GaN NW nuclei grown on Al-polar AlN surfaces did not match the polarity of the surrounding AlN surfaces. Instead, these NWs nucleated on inversion domains in the AlN layer, as revealed by cross-section STEM images, and were observed to propagate into the NW nuclei. PFM measurements suggest that these NWs are predominantly N-polar and that spontaneous nucleation of NWs is in general biased towards N-polarity. PFM has been shown to be a useful and convenient technique for mapping the overall polarity of a statistically significant number of GaN NW nuclei.

\section{Acknowledgements}

Financial support for this research was provided by the National Institute of Standards and Technology (NIST).

\section{References}

${ }^{1}$ Colin Wood and Debdeep Jena, Polarization effects in semiconductors: from ab initio theory to device applications. (Springer Science \& Business Media, 2007).

2 Matt D. Brubaker, Shannon M. Duff, Todd E. Harvey, Paul T. Blanchard, Alexana Roshko, Aric W. Sanders, Norman A. Sanford, and Kris A. Bertness, Cryst. Growth Des. 16 (2), 596 (2016).

3 A. J. Ptak, M. R. Millecchia, T. H. Myers, K. S. Ziemer, and C. D. Stinespring, Appl. Phys. Lett. 74 (25), 3836 (1999).

${ }^{4}$ B. L. VanMil, Huicheng Guo, L. J. Holbert, Kyoungnae Lee, T. H. Myers, Ting Liu, and D. Korakakis, J. Vac. Sci. Technol. B 22 (4), 2149 (2004).

5 A. J. Ptak, T. H. Myers, L. T. Romano, C. G. Van de Walle, and J. E. Northrup, Appl. Phys. Lett. 78 (3), 285 (2001).

${ }^{6}$ F. Tuomisto, K. Saarinen, B. Lucznik, I. Grzegory, H. Teisseyre, T. Suski, S. Porowski, P. R. Hageman, and J. Likonen, Appl. Phys. Lett. 86 (3), 031915 (2005).

${ }^{7}$ Uwe Karrer, Oliver Ambacher, and Martin Stutzmann, Appl. Phys. Lett. 77 (13), 2012 (2000).

${ }^{8}$ Joon Seop Kwak, K. Y. Lee, J. Y. Han, J. Cho, S. Chae, O. H. Nam, and Y. Park, Appl. Phys. Lett. 79 (20), 3254 (2001).

9 T. Auzelle, B. Haas, A. Minj, C. Bougerol, J. L. Rouviere, A. Cros, J. Colchero, and B. Daudin, J. Appl. Phys. 117 (24) (2015).

${ }^{10}$ Matt D. Brubaker, Igor Levin, Albert V. Davydov, Devin M. Rourke, Norman A. Sanford, Victor M. Bright, and Kris A. Bertness, J. Appl. Phys. 110 (5), 053506 (2011).

${ }^{11}$ Sergio Fernández-Garrido, Xiang Kong, Tobias Gotschke, Raffaella Calarco, Lutz Geelhaar, Achim Trampert, and Oliver Brandt, Nano. Lett. 12 (12), 6119 (2012).

${ }^{12}$ Ludovic Largeau, Elisabeth Galopin, Noelle Gogneau, Laurent Travers, Frank Glas, and Jean-Christophe Harmand, Cryst. Growth Des. 12 (6), 2724 (2012).

${ }^{13}$ A. Minj, A. Cros, N. Garro, J. Colchero, T. Auzelle, and B. Daudin, Nano. Lett. (2015).

${ }^{14}$ E. Soergel, J Phys D Appl Phys 44 (46), 464003 (2011).

${ }^{15}$ B. J. Rodriguez, A. Gruverman, A. I. Kingon, and R. J. Nemanich, J. Cryst. Growth 246 (3-4), 252 (2002).

${ }^{16}$ B. J. Rodriguez, A. Gruverman, A. I. Kingon, R. J. Nemanich, and O. Ambacher, Appl. Phys. Lett. 80 (22), 4166 (2002).

${ }^{17}$ R. Calarco, R. Meijers, T. Stoica, and H. Luth, Phys Status Solidi A 202 (5), 785 (2005).

${ }^{18}$ Kris A. Bertness, Matt D. Brubaker, Todd E. Harvey, Shannon M. Duff, Aric W. Sanders, and Norman A. Sanford, Phys. Status Solidi C 11 (3-4), 590 (2014). 
${ }^{19}$ G. Koblmueller, R. Averbeck, L. Geelhaar, H. Riechert, W. Hosler, and P. Pongratz, J. Appl. Phys. 93 (12), 9591 (2003).

${ }^{20}$ Karine Hestroffer, Cédric Leclere, Catherine Bougerol, Hubert Renevier, and Bruno Daudin, Phys. Rev. B 84 (24), 245302 (2011).

${ }^{21}$ O. Romanyuk, S. Fernández-Garrido, P. Jiříček, I. Bartoš, L. Geelhaar, O. Brandt, and T. Paskova, Appl. Phys. Lett. 106 (2), 021602 (2015). 


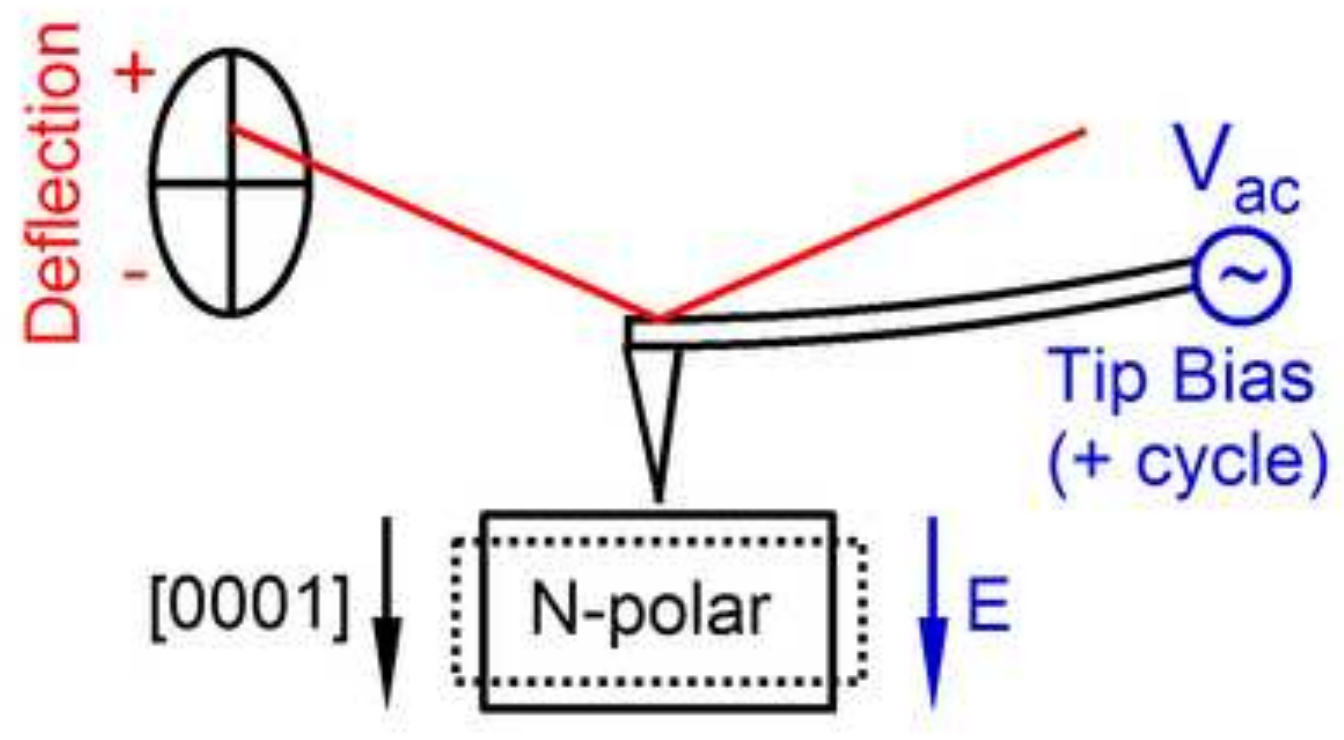

(a)

$$
\begin{gathered}
\varepsilon_{3}=d_{33} \cdot E_{3} \\
++++
\end{gathered}
$$

Tip Bias / Deflection $+$ In-phase

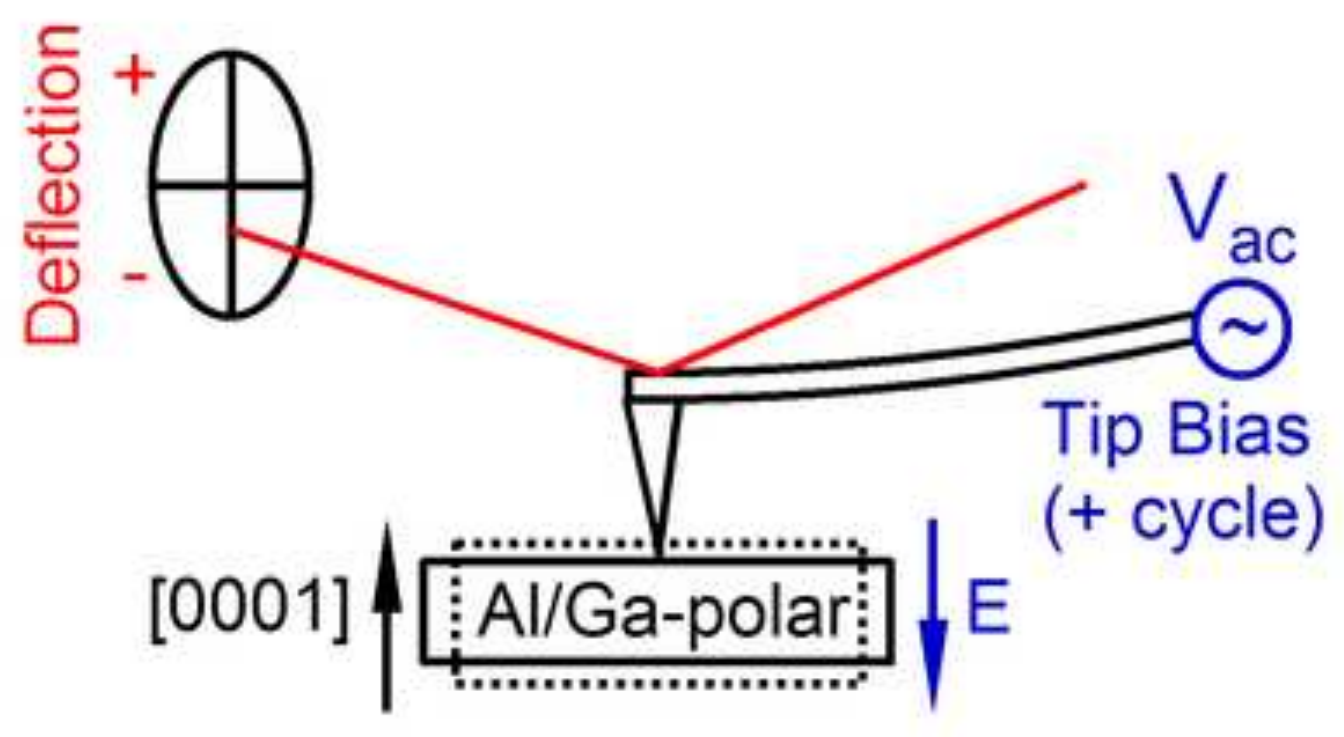

(b)

$$
\begin{gathered}
\varepsilon_{3}=d_{33} \cdot E_{3} \\
-+\quad+
\end{gathered}
$$

Tip Bias / Deflection $+$ Out-of-phase 


\section{Figure 3}

Click here to download high resolution image

(a)

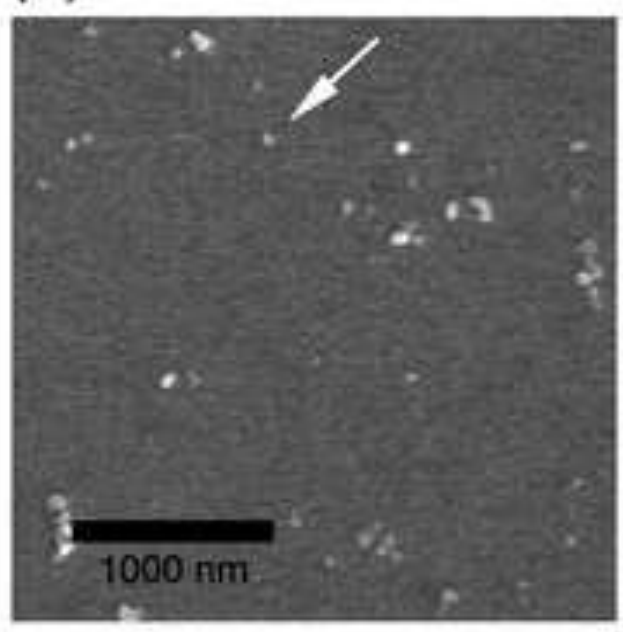

O uV $\quad 75$ uV $150 u \mathrm{~V}$ (c) PFM Amplitude

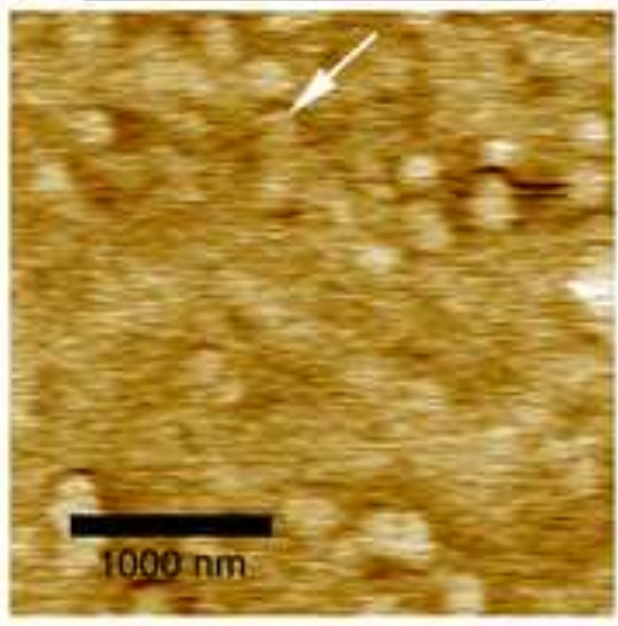

(e)

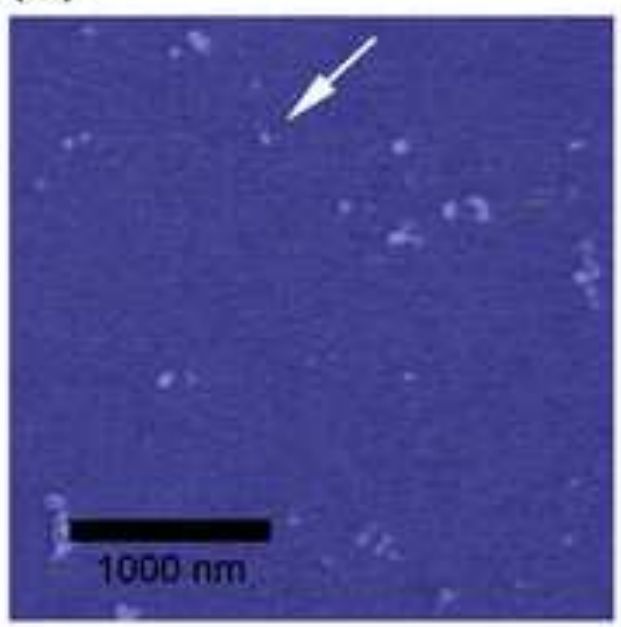

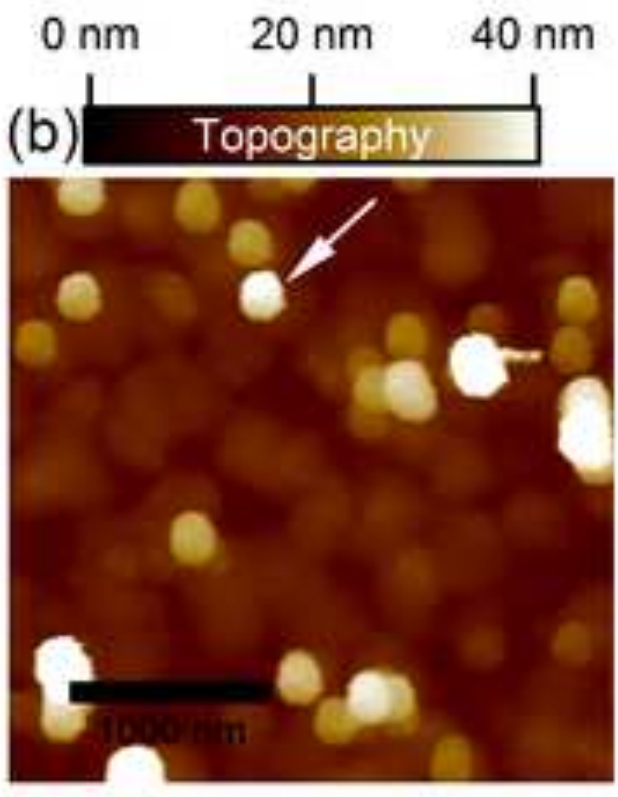

$\begin{array}{ll}\text { Al/Ga-polar } & N \text {-polar } \\ -180 \mathrm{deg}-90 \mathrm{deg} & 0 \mathrm{deg}\end{array}$
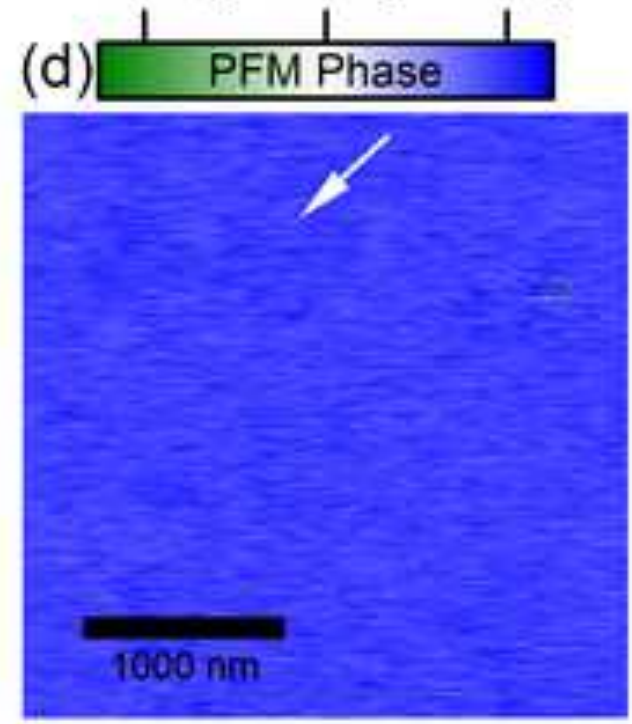

(f)

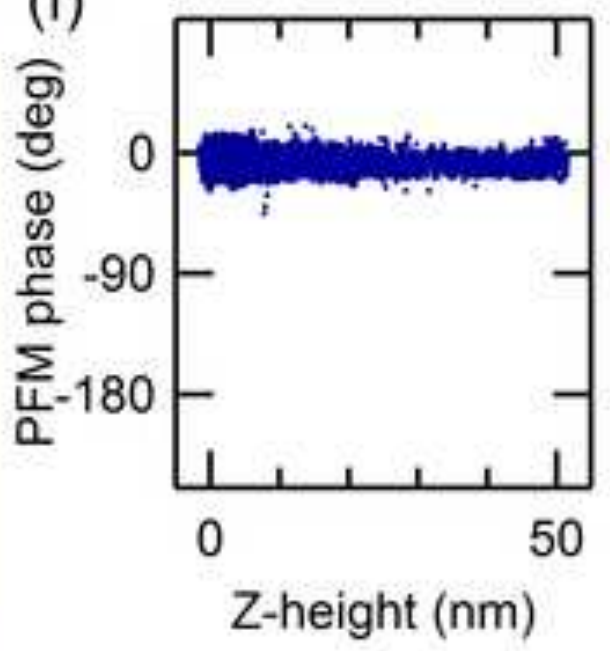


(a)

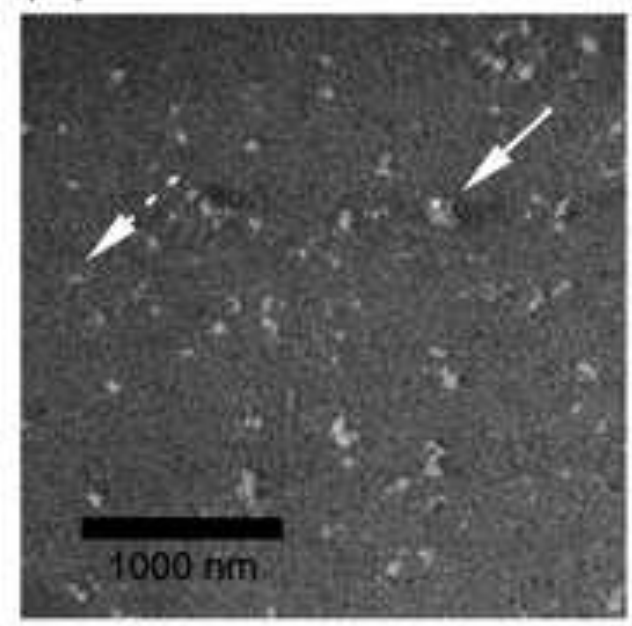

o uV 75 uV
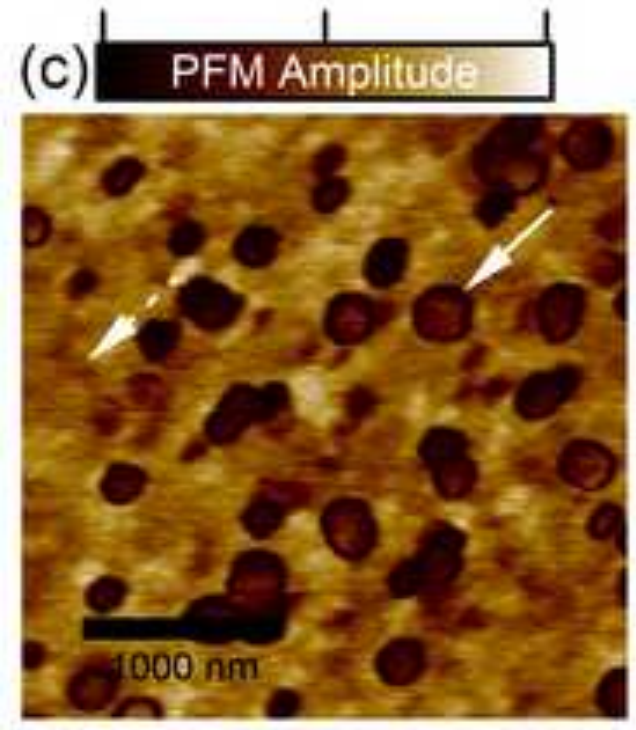

(e)

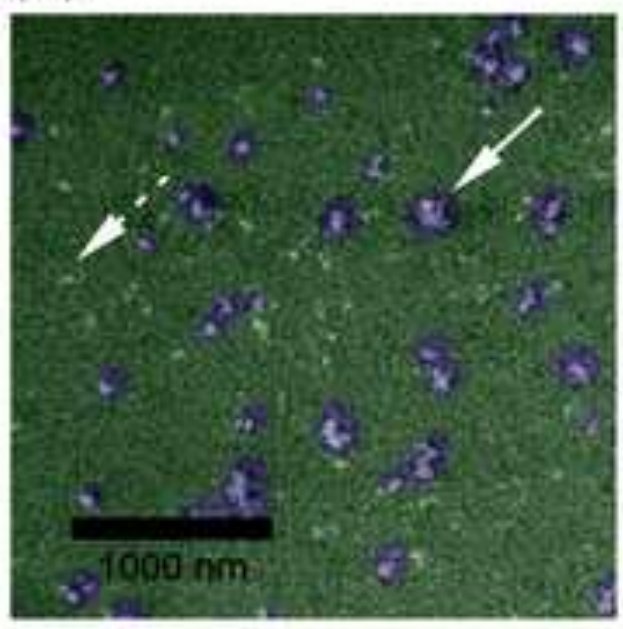

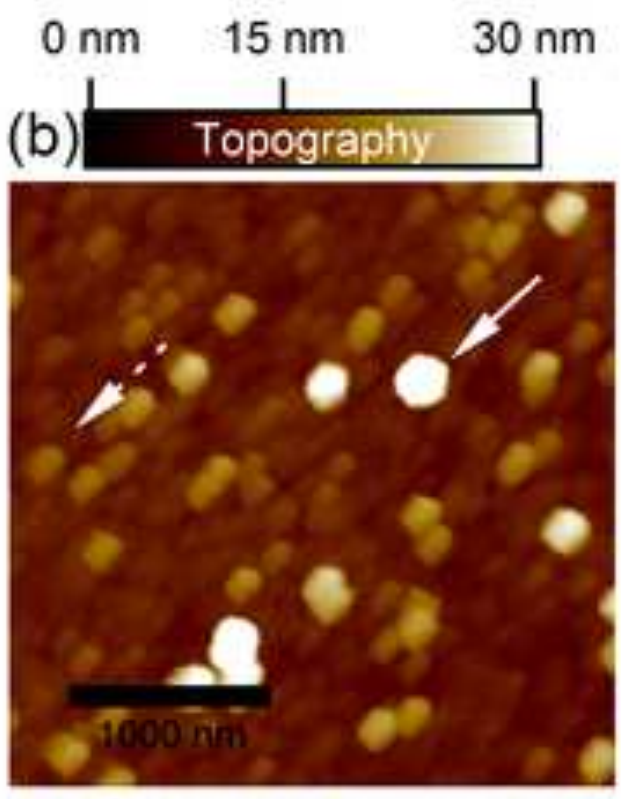

$\begin{array}{ll}\text { Al/Ga-polar } & \text { N-polar } \\ -180 \mathrm{deg}-90 \mathrm{deg} & 0 \mathrm{deg}\end{array}$
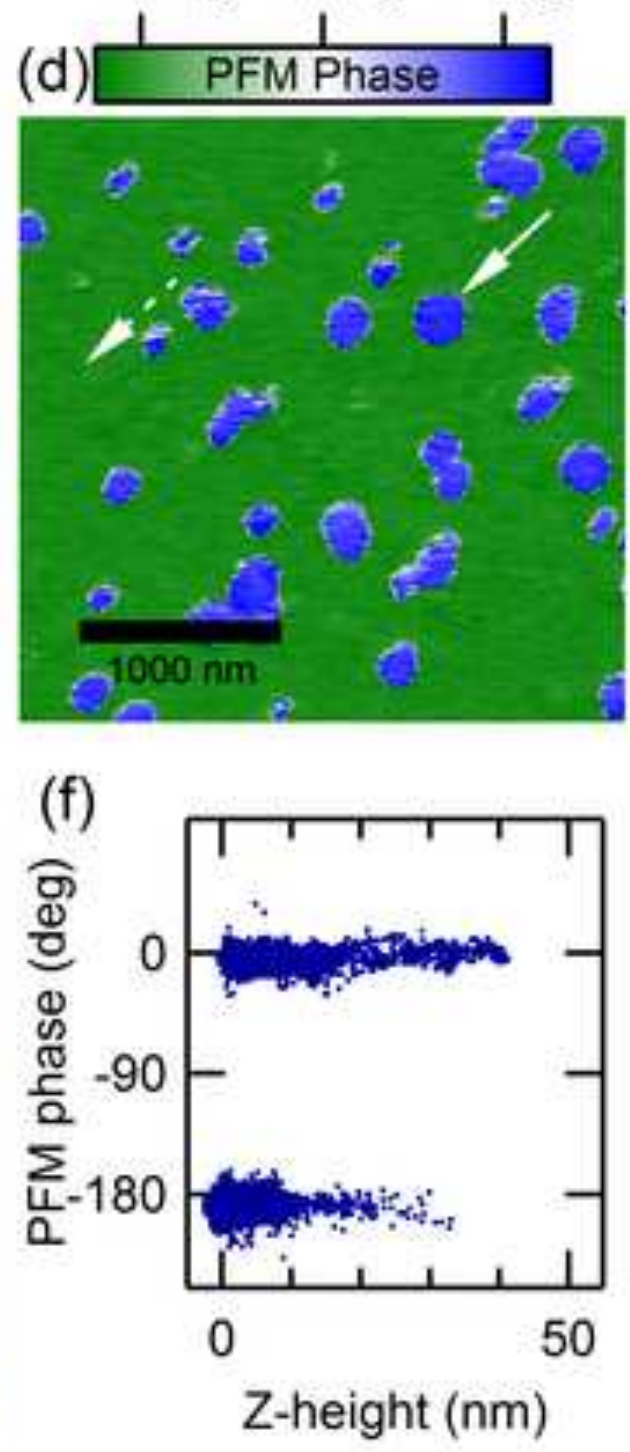
Click here to download high resolution image

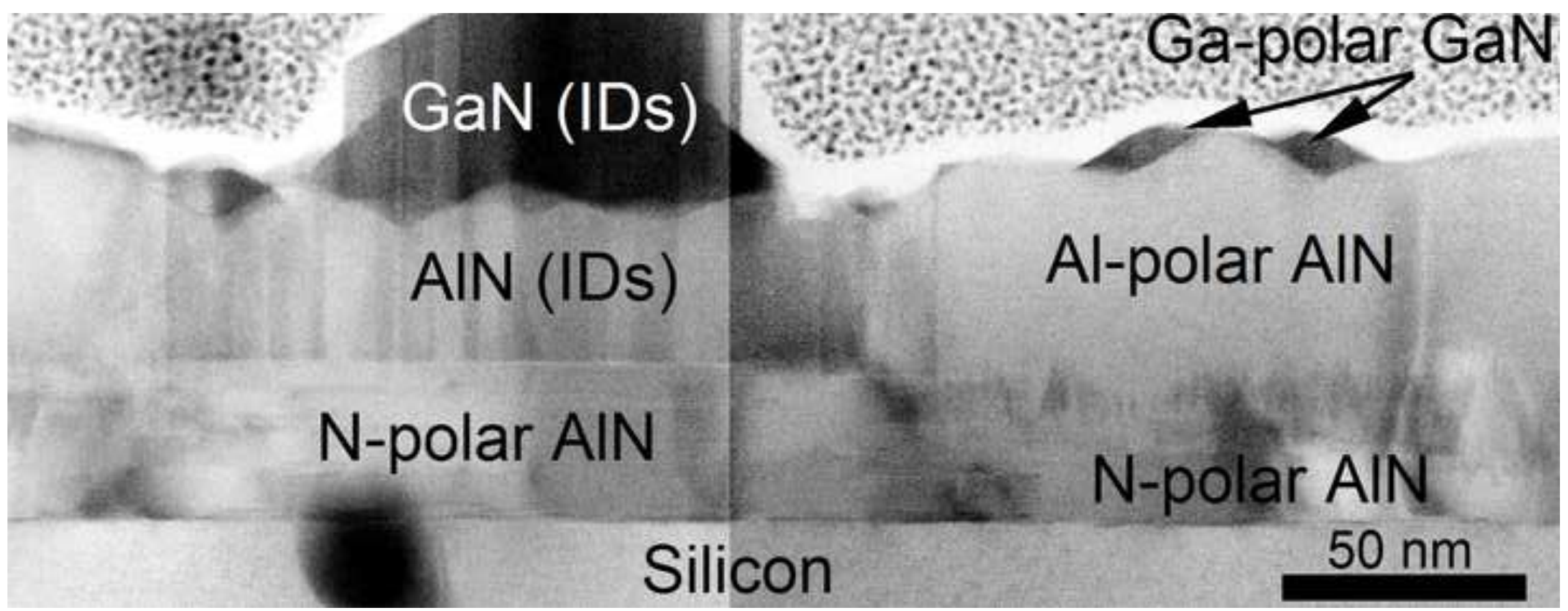

\title{
GaN (IDs)
}

Al-polar AIN

\author{
N-polar AIN
}

Silicon

N-polar AIN

Gapolar GaN

AIN (IDs) 